\title{
Impactación cubitocarpiana
}

\author{
Gustavo J. Teruya, Nicolás N. Altamirano \\ Unidad de Cirugía del Miembro Superior, Servicio de Ortopedia y Traumatología, Hospital Británico de Buenos Aires, \\ Ciudad Autónoma de Buenos Aires, Argentina
}

\section{RESUMEN}

El objetivo de este artículo es actualizar los conocimientos sobre la impactación cubitocarpiana y su tratamiento. Estudios clásicos sobre la biomecánica del borde cubital de la muñeca han demostrado que los cambios milimétricos en la relación de longitud entre el cúbito y el radio alteran significativamente la transferencia de cargas entre los huesos del carpo, el radio y el cúbito. Así, un aumento relativo en la longitud del cúbito generará una carga excesiva sobre la articulación cubitocarpiana que producirá un espectro de cambios degenerativos progresivos en el domo cubital, el semilunar, el piramidal y el complejo del fibrocartílago triangular que finalizarán con artrosis cubitocarpiana y radiocubital distal. La impactación cubitocarpiana, en sus diversos estadios degenerativos, se puede tratar mediante osteotomías que buscan descomprimir la carga cubitocarpiana. Las osteotomías pueden ser extrarticulares o intrarticulares. Entre las extrarticulares, están las diafisarias, las metafisarias sin exposición articular (subcapitales) y las metafisarias distales con exposición articular y, entre las intrarticulares, la cirugía de resección en oblea (wafer), que reseca cartílago y hueso subcondral del domo cubital, y puede ser un procedimiento abierto o artroscópico. Si hay artrosis radiocubital distal, solo se podrá tratar con cirugías de rescate, como Darrach, Sauvé-Kapandji, Bowers, o una prótesis radiocubital distal. Estas técnicas de osteotomía se han analizado detalladamente para lograr definir sus ventajas y desventajas. Finalmente se propone una forma de tipificar la impactación cubitocarpiana, cuyo objetivo es orientar al lector hacia el mejor tratamiento posible, avalado por la bibliografía actual.

Palabras clave: Impactación cubitocarpiana; cúbito plus; osteotomía; acortamiento; procedimiento tipo wafer.

Nivel de Evidencia: V

\section{Ulnocarpal Impaction}

\section{ABSTRACT}

The purpose of this article is to provide updated knowledge about ulnocarpal impaction syndrome (UCIS) and its treatment. Classic studies on biomechanics of the ulnar side of the wrist have shown that millimetrical changes in the relative lengths of the ulna and the radius significantly alter the load transmission between the carpal bones, the radius and the ulna. Thus, an increase in the relative length of the ulna will generate an excessive load on the ulnocarpal joint, which will produce a spectrum of progressive degenerative changes in the ulnar dome, lunate, triquetrum, and the triangular fibrocartilage complex (TFCC), that will lead to ulnocarpal and distal radioulnar joint (DRUJ) osteoarthritis. In its various degenerative stages, UCIS can be treated with osteotomies that seek to decompress the ulnocarpal load. These can be extra-articular or intra-articular. Within the extra-articular osteotomies, we find the diaphyseal, metaphyseal without joint exposure (subcapital), and the distal metaphyseal with joint exposure. Within the intra-articular ones, we find the wafer procedure, which resects the cartilage and subchondral bone of the ulnar dome, and can be performed either openly or arthroscopically. If there is associated DRUJ osteoarthritis, it can only be treated with salvage surgeries such as the Darrach, Sauvé-Kapandji, and Bowers procedures, or a DRUJ arthroplasty. These osteotomy techniques will be analyzed in detail in order to define their advantages and disadvantages. Finally, we propose a way to typify the UCIS to guide the reader towards the best possible treatment supported by current literature.

Keywords: Ulnocarpal impactation syndrome; positive variance; osteotomy; shortening; wafer.

Level of Evidence: $\mathrm{V}$ 


\section{INTRODUCCIÓN}

El objetivo de este artículo es presentar una revisión bibliográfica de las publicaciones en inglés sobre el síndrome de impactación cubitocarpiana (ICC), desde los clásicos hasta hoy, a fin de actualizar el conocimiento y generar una guía para la elección del tratamiento.

\section{BIOMECÁNICA}

Una de las características de la evolución, desde los primates hasta el hombre, es el aumento del rango y la precisión en la movilidad de la muñeca y la mano. En la mano, está dado por la oposición del pulgar y, en la muñeca, es el resultado de dos modificaciones esqueléticas: el cúbito distal se aleja del piramidal y el pisiforme, y comienza a articular con el radio distal aumentando el desvío cubital y la pronosupinación. De esta forma, la evolución de la articulación radiocubital distal $(\mathrm{RCD})$ y cubitocarpiana en el ser humano permite un marcado rango de movilidad del antebrazo y la muñeca, sin interferir con la función de agarre de la mano. ${ }^{1}$ Durante el movimiento de pronosupinación, el radio y el carpo giran alrededor de un eje que es el cúbito y, teniendo en cuenta la relación RCD, la cabeza del cúbito se desplaza hacia dorsal y distal en pronación, y hacia volar y proximal en supinación.

En 1984, Palmer y Werner demostraron que normalmente el radio recibe el $82 \%$ de la carga axial transmitida desde el carpo, y el cúbito, el 18\% a través del complejo del fibrocartílago triangular (FCT). Cambios milimétricos en la relación de longitud RCD alteran, en forma sustancial, la transferencia de cargas. Es así que una discrepancia de longitud de $+2,5 \mathrm{~mm}$ (cúbito plus) aumentará su carga desde el carpo al $42 \%$ y de $-2,5 \mathrm{~mm}$ (cúbito minus), la reducirá al $4 \%$. Este concepto de pequeños cambios en la longitud asociados a grandes alteraciones en las cargas es fundamental para entender la biomecánica de este cuadro y los principios de su tratamiento. ${ }^{2}$

Se ha descrito una relación directa entre el aumento de la longitud del cúbito con respecto a la del radio y la edad. Esta podría estar asociada al desgaste asimétrico del cartílago articular a nivel del codo, que es mayor en la cúpula radial. ${ }^{3}$ Por otra parte, Orbay y cols. sostienen que la elongación progresiva de la banda central de la membrana interósea sería, entre otras, la causa del cambio de la relación anatómica entre el cúbito y el radio, generando un cúbito plus adquirido por la edad. ${ }^{4}$

Harley y cols., basándose en investigaciones previas realizadas por Werner sobre la transmisión de fuerzas a través del FCT, llevaron a cabo un estudio cadavérico en el que hallaron que, cuando existe un cúbito minus constitucional, el disco articular del complejo del FCT tiene un espesor mayor y es más delgado cuando la relación es inversa (cúbito plus). De esta forma, la transmisión de fuerzas desde la muñeca hacia el antebrazo no se ve influenciada por la varianza cubital constitucional. Sostienen la hipótesis de que la mayor incidencia de ICC que se observa en la varianza cubital positiva está relacionada con un disco central más delgado y, por lo tanto, con una tendencia mayor a perforarse. ${ }^{5}$ Sin embargo, también puede presentarse en varianzas neutras e incluso negativas. Friedman y cols. han demostrado que la maniobra de fuerza de puño y pronación produce un acortamiento relativo del radio poniendo de manifiesto una ICC dinámica que, en forma repetitiva, a través del tiempo, puede generar erosión, luego perforación del disco articular y condromalacia en el cúbito, semilunar y piramidal.

\section{DEFINICIÓN}

La ICC es una enfermedad producida por una carga excesiva sobre la articulación cubitocarpiana que produce un espectro de cambios degenerativos progresivos en el extremo distal del cúbito, el semilunar, el piramidal y el complejo del FCT. ${ }^{6,7}$ Las alteraciones habituales son el desgarro degenerativo del FCT; la condromalacia en el semilunar, el piramidal y en el domo de la cabeza del cúbito; la inestabilidad o desgarro del ligamento lunopiramidal y, finalmente, la artrosis de las articulaciones cubitocarpiana y RCD. ${ }^{7,8}$ Palmer diseñó un sistema de clasificación para las lesiones del complejo del FCT basada en el mecanismo de lesión, la localización y las estructuras involucradas, que nos ayuda a orientar el manejo clínico (Tabla 1).

Cuando ocurren cambios artrósicos, habitualmente se los trata mediante procedimientos de rescate. Sin embargo, cuando la ICC se diagnostica de manera temprana, existen distintos tipos de tratamientos propuestos para detener su progresión. El objetivo principal del tratamiento es descargar el compartimento cubitocarpiano, pero también restablecer la congruencia de la articulación RCD y ajustar la tensión del complejo del FCT. ${ }^{6}$ 
Tabla 1. Clasificación de las lesiones del complejo del fibrocartílago triangular según Palmer y cols.

\begin{tabular}{|l|l|}
\hline A. Perforación central \\
\hline Blase I - Traumáticas & B. Avulsión del borde cubital \\
\hline C. Avulsión de ligamentos cubitocarpianos \\
\hline D. Avulsión del borde radial \\
\hline A. Desgaste central del complejo del fibrocartílago triangular \\
\hline B. IIA + condromalacia del semilunar, la cabeza cubital, o de ambos \\
\hline C. Perforación del complejo del fibrocartílago triangular y condromalacia del semilunar, \\
\hline la cabeza cubital, o de ambos \\
\hline D. IIC + perforación del ligamento lunopiramidal \\
\hline E. IID + artrosis cubitocarpiana
\end{tabular}

Este cuadro habitualmente se asocia a una varianza cubital positiva, ya sea congénita o adquirida. La varianza cubital se define como la diferencia de longitud relativa entre la esquina cubital de la fosa semilunar del radio y el punto más distal del domo de la cabeza cubital. Para realizar esta medición, en una radiografía anteroposterior de muñeca en rotación neutra, se trazan dos líneas perpendiculares al eje longitudinal del antebrazo, tangenciales al extremo proximal de la fosa semilunar del radio y al extremo distal de la superficie articular del domo del cúbito (Figura 1). ${ }^{9}$ Es positiva cuando el domo cubital está distal al borde medial de la fosa semilunar. ${ }^{8,10}$ El valor se expresa en milímetros y es independiente de la longitud de la apófisis estiloides cubital. ${ }^{8}$

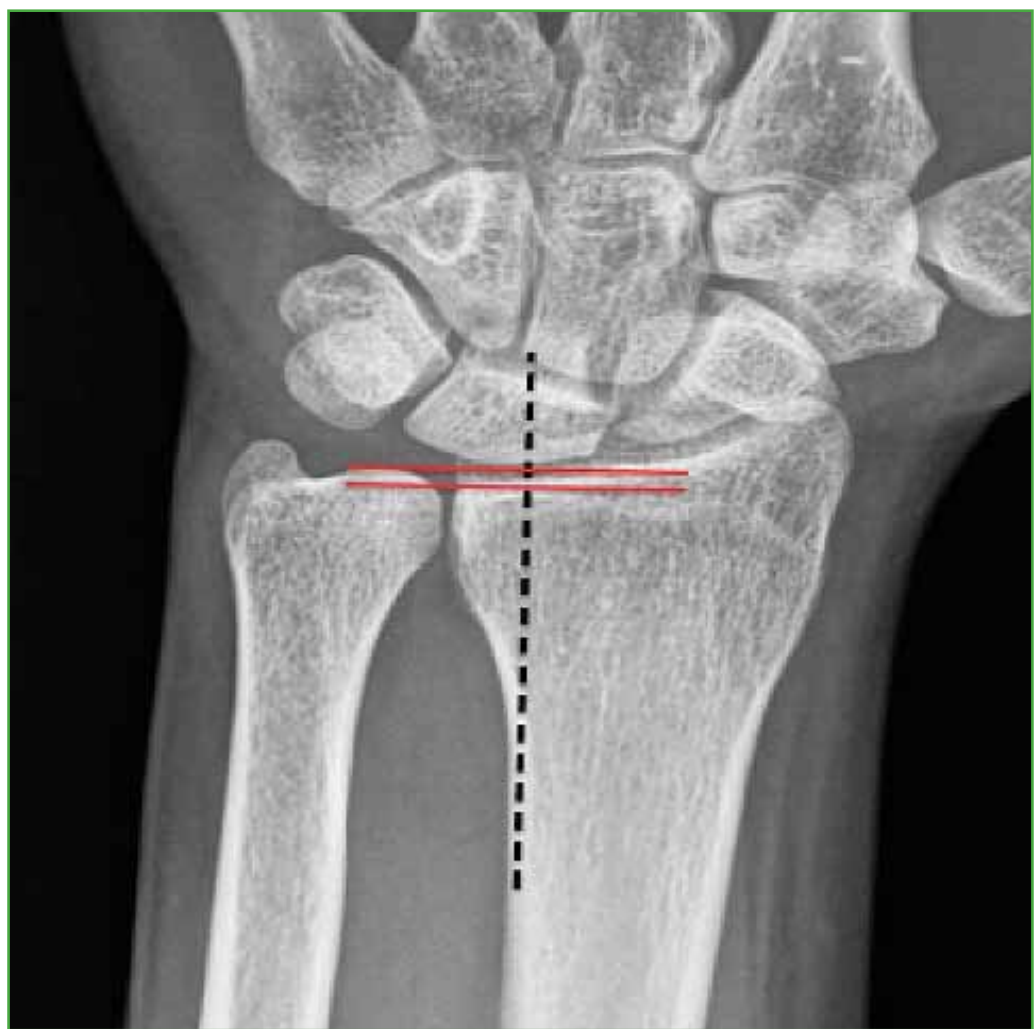

Figura 1. Medición de la discrepancia radiocubital distal en la radiografía de muñeca 
La sobrecarga entre el carpo y la cabeza del cúbito cursa con dolor en el borde cubital de la muñeca y limitaciones en la desviación cubital y la pronosupinación. Junto a los cambios degenerativos ya mencionados, puede haber una inestabilidad de la articulación RCD debido a lesiones ligamentarias posteriores a la rotura o avulsión del complejo del FCT. ${ }^{10}$ Suele ocurrir en casos de consolidación viciosa del radio distal, escisión de la cúpula radial, cierre prematuro de la fisis del radio, lesión de Essex-Lopresti, varianza cubital positiva congénita, deformidad de Madelung o en cualquier cuadro que cause un incremento en la longitud relativa del cúbito. ${ }^{11}$

Como ya se mencionó en la sección de biomecánica, Friedman y Palmer demostraron que la ICC puede ser un cuadro dinámico, debido a los cambios intermitentes en la varianza cubital con las actividades de la vida cotidiana, en ocupaciones que requieren movimientos de prensión repetitiva y pronación del antebrazo. Así el cierre de puño con fuerza máxima en pronación resulta en un incremento en promedio de 1,95 $\mathrm{mm}$ en la varianza cubital. Por lo tanto, ciertas actividades repetitivas generarían aumentos de la carga cubital del carpo, desarrollando cambios secundarios a largo plazo.

\section{PRESENTACIÓN CLÍNICA Y DIAGNÓSTICO}

El cuadro clínico suele tener un comienzo insidioso y progresivo. Los pacientes refieren sentir dolor en el borde cubital de la muñeca, con episodios ocasionales de edema y disminución de la movilidad de la muñeca. Estos síntomas se pueden exacerbar durante las actividades que involucren el cierre de puño con fuerza, la pronación o la desviación cubital, y se alivian con el reposo.

El diagnóstico del síndrome de ICC se basa principalmente en el examen físico, y se complementa con estudios por imágenes. ${ }^{12}$ Se debe realizar una evaluación minuciosa para descartar otras fuentes de dolor en el borde cubital de la muñeca, como artritis pisipiramidal, artrosis de la articulación RCD, tendinitis del cubital posterior (ECU) o neuritis de la rama cutáneo dorsal del nervio cubital, entre otras.

La maniobra de estrés cubitocarpiano fue descrita por Nakamura y cols. ${ }^{13}$ con el paciente sentado, su codo apoyado sobre la mesa y el antebrazo en rotación neutra, se coloca la muñeca en la máxima desviación cubital y, mientras se aplica una carga axial, se rota en pronosupinación. Esta prueba se considera positiva cuando reproduce los síntomas dolorosos por los que consulta el paciente (Figura 2).

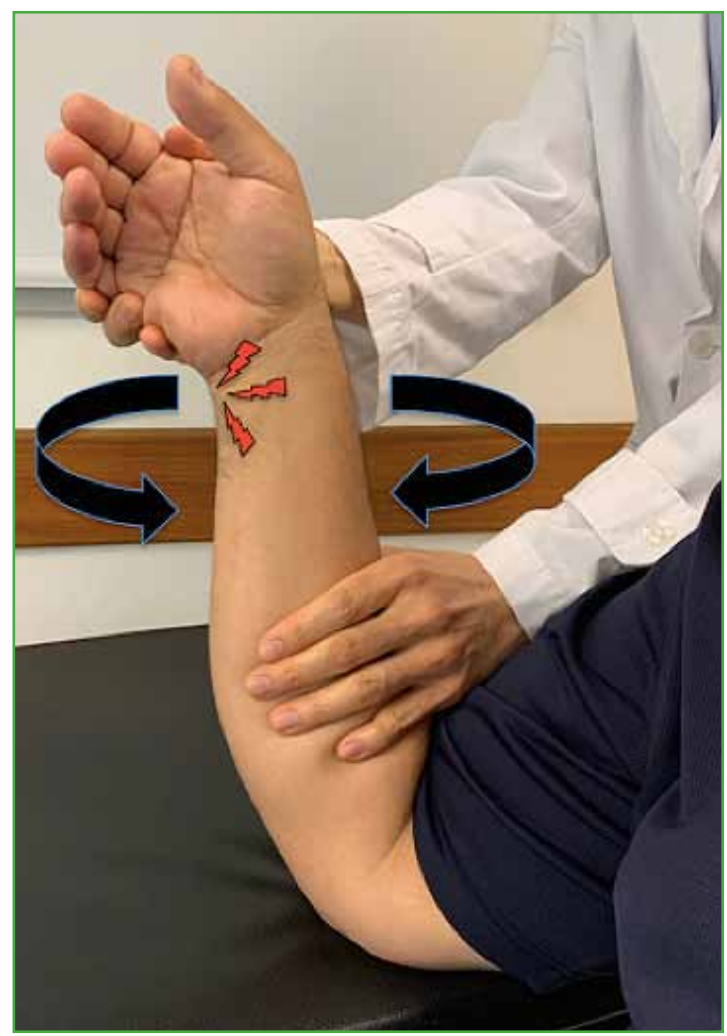

Figura 2. Maniobra de Nakamura. 
El signo de la fóvea también suele ser positivo, aunque no es específico de la ICC. Consiste en la aparición de dolor al presionar con el pulgar el punto blando entre la apófisis estiloides cubital, el tendón del cubital anterior (FCU) y el hueso pisiforme. Si el ligamento lunopiramidal está comprometido, las maniobras de provocación, como las pruebas de Reagan y de Kleinman, pueden ser positivas. Finalmente, es importante también evaluar la articulación RCD.

\section{Estudios por imágenes}

Ante la sospecha de ICC, el primer estudio que se debe solicitar es una radiografía comparativa anteroposterior de muñeca. Se realiza con el hombro en abducción de $90^{\circ}$, el codo flexionado a $90^{\circ}$ y el antebrazo en rotación neutra. También debe tomarse una radiografía de perfil y otra de frente en pronación máxima con fuerza de puño. ${ }^{8}$ Así se evalúa la varianza cubital tanto estática como dinámica.

Las radiografías características pueden mostrar esclerosis subcondral o cambios quísticos en el domo del cúbito, el borde posteromedial del semilunar o el borde posterolateral del piramidal. ${ }^{8}$ En casos severos, se puede observar una franca artrosis con formación osteofitaria en la articulación cubitocarpiana. Si se evalúa la posibilidad de realizar una osteotomía de acortamiento, también debe examinarse la articulación RCD y su inclinación anatómica. En el perfil, se debe buscar si hay una subluxación dorsal del cúbito. ${ }^{14}$

Ante una alta sospecha clínica de ICC, pero sin signos radiográficos, la resonancia magnética es de suma utilidad. En los estadios tempranos, puede observarse fibrilación a nivel del cartílago articular del carpo y de la cabeza del cúbito. El cuadro progresa a edema óseo (Figura 3) y luego a cambios escleróticos. ${ }^{8}$ En pacientes con signos radiográficos de ICC, la artrorresonancia es útil para evaluar la integridad del FCT y del ligamento lunopiramidal. ${ }^{7,9}$

El síndrome de ICC debe diferenciarse de otros cuadros que afectan el semilunar. Los cambios radiológicos en la estructura del semilunar son fundamentales para distinguir otras etiologías, como la enfermedad de Kienböck o los quistes intraóseos. En el primer caso, las imágenes son más difusas, afectan la mitad radial del semilunar y no hay compromiso del piramidal ni del domo del cúbito. Los quistes intraóseos aparecen como una imagen de bordes definidos, sin afectar huesos vecinos. ${ }^{8}$

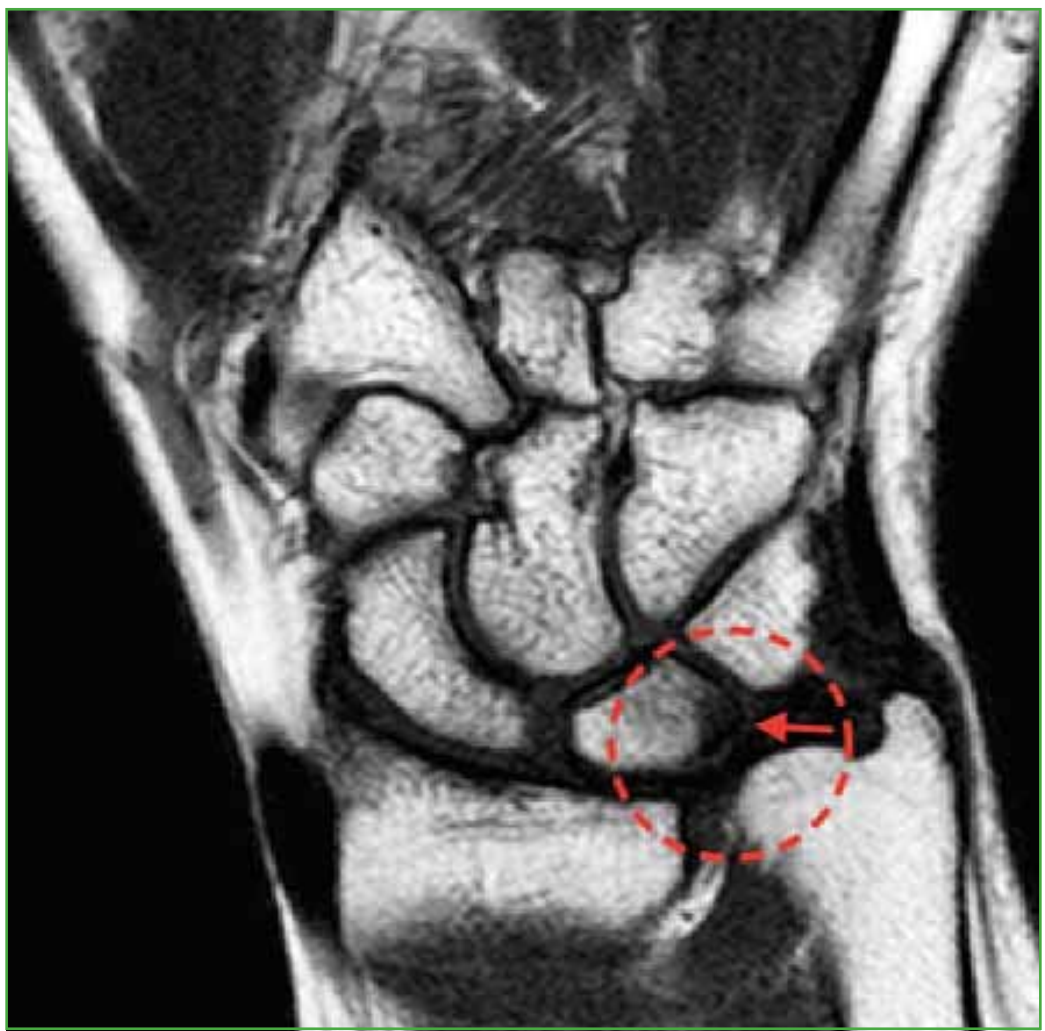

Figura 3. Resonancia magnética de muñeca con impactación cubitocarpiana. Edema en el semilunar. 


\section{TRATAMIENTO}

Hay acuerdo en que el síndrome de ICC, sobre todo en los casos idiopáticos, se debe tratar inicialmente con recursos no quirúrgicos entre tres y seis meses. Esto incluye férulas de inmovilización, modificación de las actividades cotidianas, antinflamatorios no esteroides, infiltraciones locales con corticoides y fisioterapia.

Si los síntomas no mejoran, se indica el tratamiento quirúrgico, cuyo objetivo principal es descargar la articulación cubitocarpiana, acortando la longitud del cúbito con respecto al radio y, de esta forma, disminuyendo la presión en el disco articular del FCT.

Si la causa fuese la consolidación viciosa de una fractura del radio distal en el plano sagital, debemos considerar una osteotomía correctiva del radio. ${ }^{15}$

La articulación cubitocarpiana se puede descargar mediante osteotomías de acortamiento extrarticulares o intrarticulares. Dentro de las extrarticulares, tenemos las diafisarias, las metafisarias sin exposición articular (subcapitales) y las metafisarias distales con exposición articular. Entre las osteotomías intrarticulares, se encuentra la cirugía descrita por Feldon, en 1992, la cual se popularizó como procedimiento de resección en oblea (wafer), que reseca cartílago y hueso subcondral del domo cubital. Puede ser un procedimiento abierto o artroscópico. Si la ICC coexiste con artrosis en la articulación RCD, la indicación es una cirugía de rescate que anula o reemplaza con una prótesis esta última articulación. Estos procedimientos son las cirugías de Darrach o Bowers como resectivas, la de Sauvé-Kapandji como artrodesis o la prótesis de cúbito distal en sus diferentes variantes (Figuras 4 y 5). ${ }^{10}$
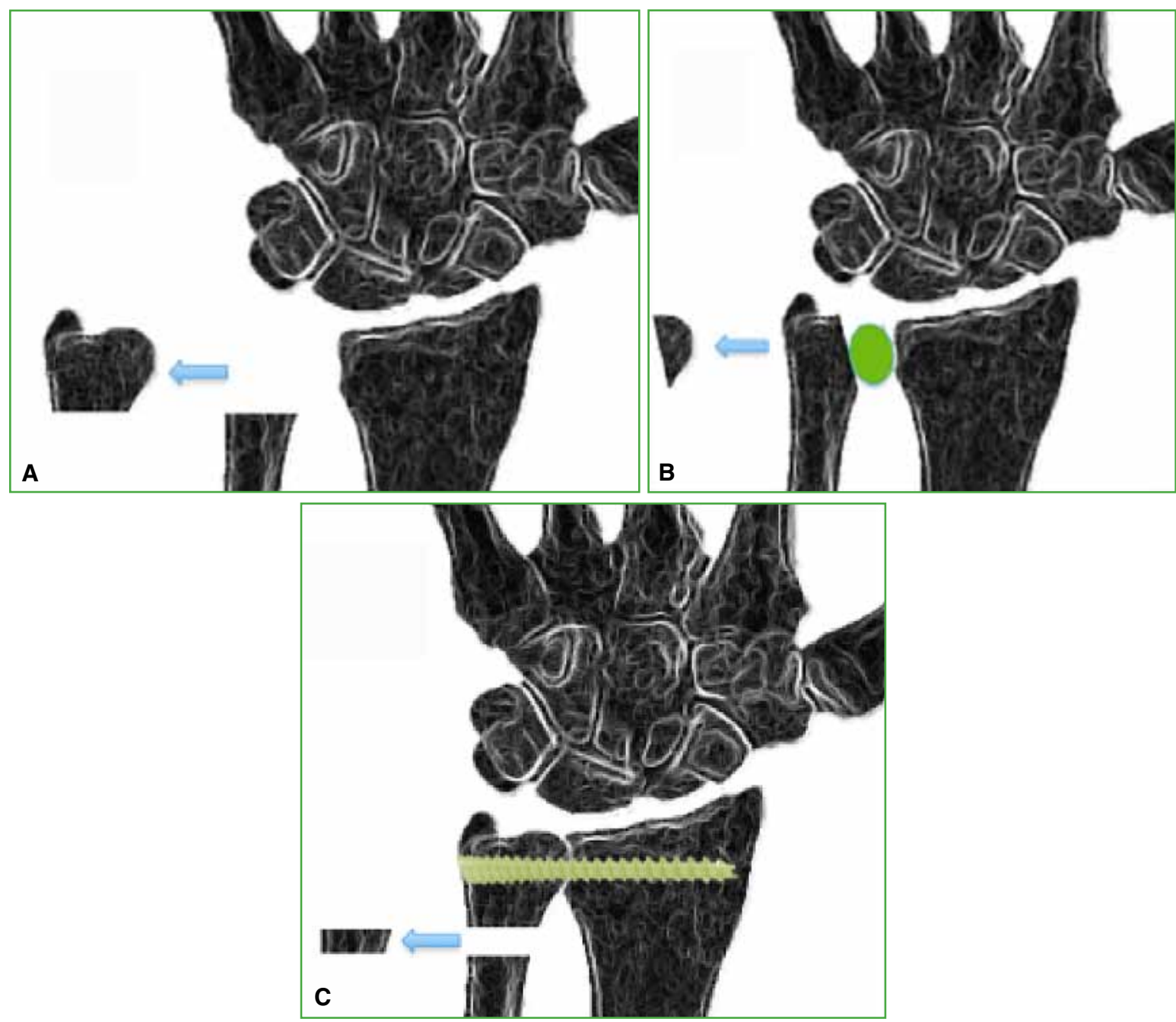

Figura 4. A. Resección de Darrach. B. Resección de Bowers. C. Sauvé-Kapandji. 


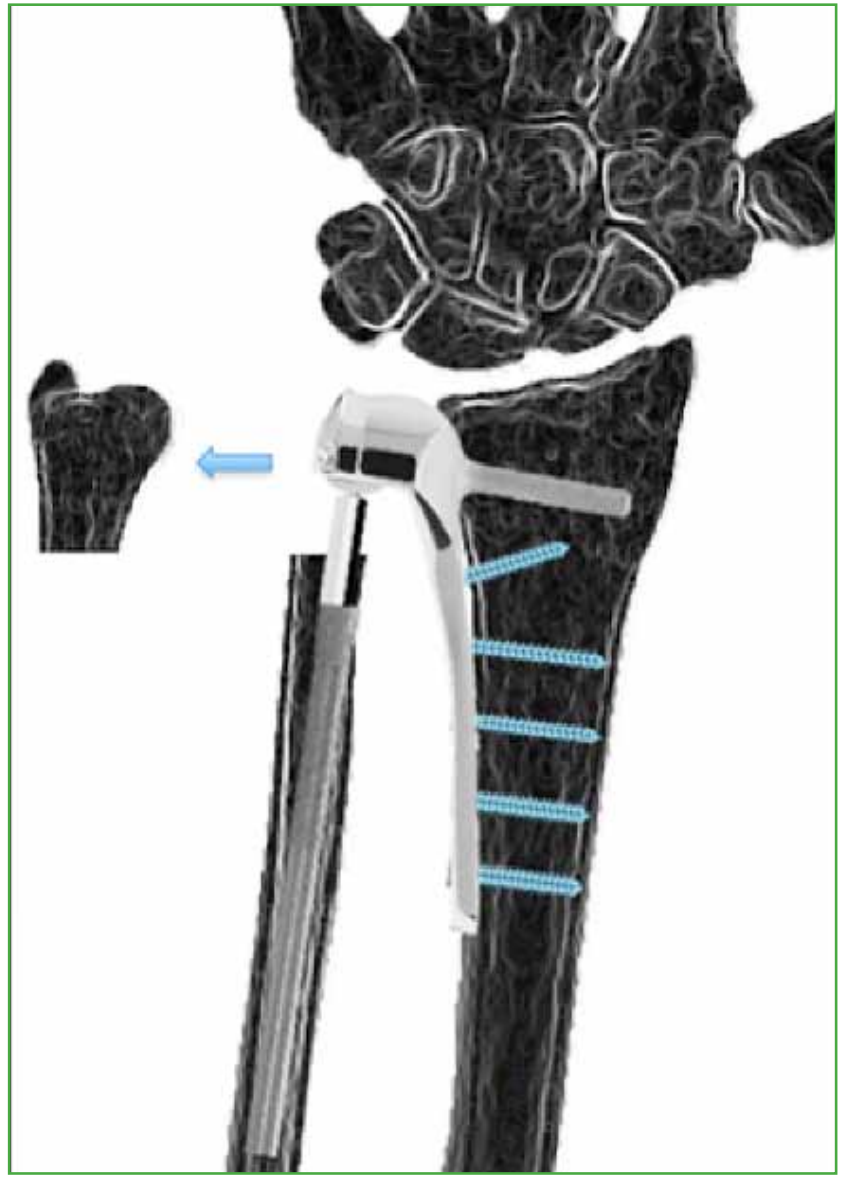

Figura 5. Prótesis de Scheker.

\section{Osteotomías diafisarias}

Milch describió, por primera vez, una osteotomía diafisaria de acortamiento cubital en 1941. Realizó este procedimiento en un paciente con una ICC como secuela de la consolidación viciosa de una fractura de radio distal.

El concepto de acortamiento cubital perduró a través del tiempo, fue mejorando la precisión de los sistemas de acortamiento y osteosíntesis, y hoy en día, se considera el patrón de referencia en el tratamiento de esta enfermedad. ${ }^{10,16}$ La primera fijación de una osteotomía transversal de acortamiento con placa y tornillos fue descrita por Cantero, en 1974 (Figura 6A). Posteriormente se describieron osteotomías oblicuas que permitieron colocar un tornillo compresivo transosteotomía con el fin de mejorar la estabilidad del sistema y la tasa de consolidación (Figura 6B).

Darlis describió una osteotomía de acortamiento diafisaria escalonada cuya ejecución es bastante más compleja, pero con las ventajas de poder colocar un tornillo compresivo transosteotomía, una mayor superficie de contacto y un mejor control de la rotación. Utilizando una placa de neutralización de $3,5 \mathrm{~mm}$, de ubicación volar, consiguió un 100\% de consolidación en su serie de 29 pacientes (Figura 6C).

El objetivo de la osteotomía de acortamiento en un cúbito plus es conseguir una varianza 0 o $-1 \mathrm{~mm}$. Ante una impactación con cúbito neutro o minus, la magnitud del acortamiento será determinada por las radiografías dinámicas en pronación y con fuerza de puño.

Minami y Kato habían notado que algunos pacientes con lesión del FCT luego del desbridamiento artroscópico continuaban con dolor. El factor común era una varianza cubital positiva que podía estar asociada a una lesión ligamentaria lunopiramidal. Por esta razón, propusieron tratar a las lesiones del FCT con cúbito plus mediante una osteotomía de acortamiento cubital. ${ }^{9}$ 

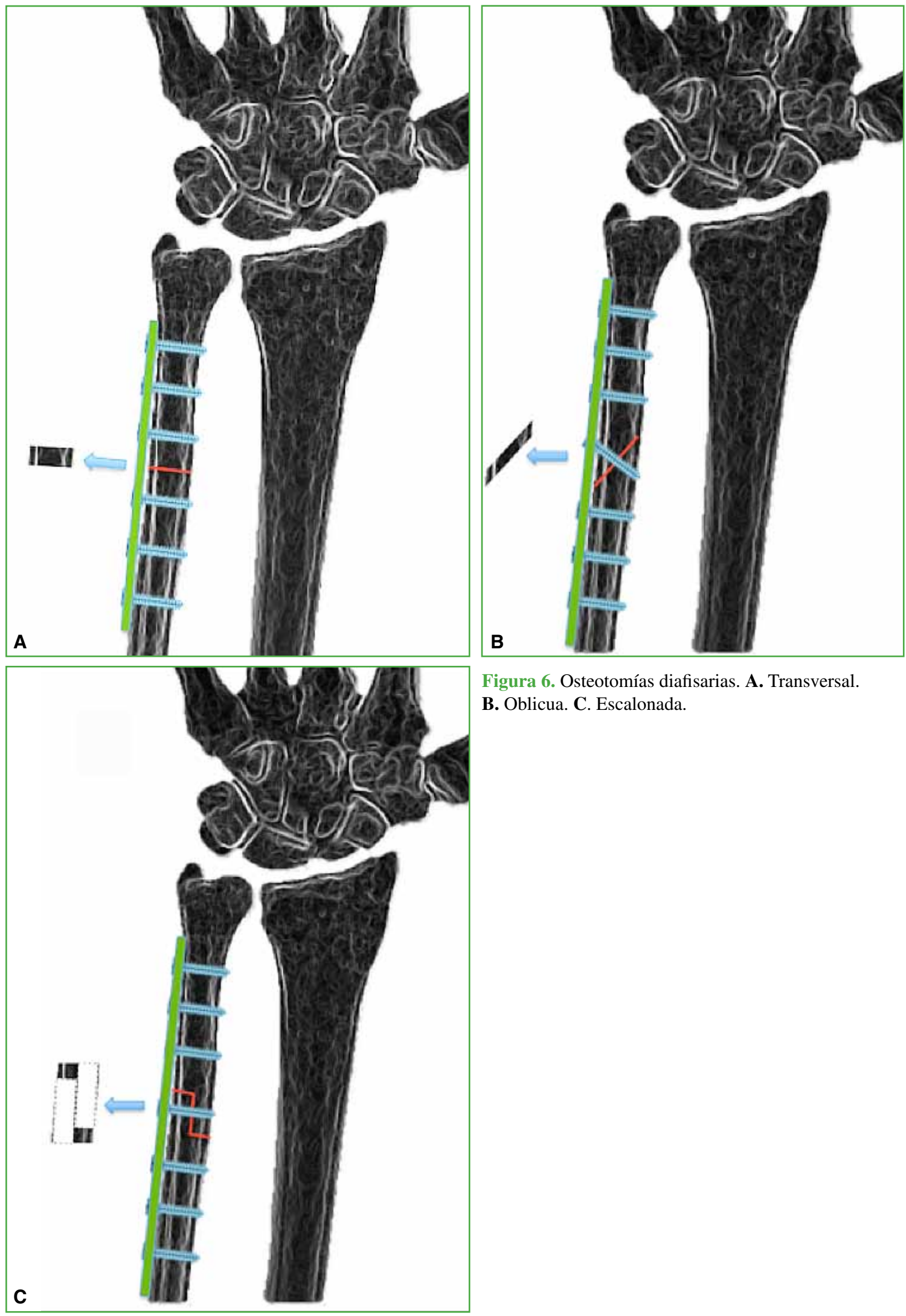

Figura 6. Osteotomías diafisarias. A. Transversal. B. Oblicua. C. Escalonada. 
En una serie de 31 pacientes, Baek y cols. hallaron una correlación positiva entre la magnitud de la varianza cubital y una subluxación dorsal de la articulación RCD. En estos casos, la cirugía de acortamiento tiende a corregir dicha subluxación. Esto estaría relacionado con el retensado de los ligamentos cubitocarpianos. ${ }^{14}$ Este mismo autor también observó que un 16\% de los pacientes sometidos a osteotomías de acortamiento tiende a desarrollar cambios artrósicos en la articulación RCD a largo plazo. Dichos cambios suelen aparecer luego de los tres años de la cirugía y se vincularían con la magnitud de la discrepancia y la subluxación dorsal radiocubital preoperatoria. Sin embargo, la aparición de artrosis no se asoció con dolor ni otros cambios clínicos y funcionales. Otros autores observaron los cambios artrósicos en el $38 \% .^{17}$

El complejo del FCT puede ser interpretado como una estructura tridimensional que estabiliza todo el borde cubital de la muñeca. Al realizar un acortamiento cubital, se produce una tracción de toda esta estructura generando mayor tensión ligamentaria y, por lo tanto, estabilizando la articulación RCD y cubitocarpiana. En un estudio biomecánico cadavérico, Nishiwaki y cols. demostraron que cuanto mayor es la magnitud del acortamiento, mayor es el efecto estabilizador si la inserción foveal estaba intacta o solo parcialmente lesionada. Cuantificaron que, para una lesión parcial, un acortamiento de $3 \mathrm{~mm}$ recupera la estabilidad original; en cambio, si existe una desinserción foveal completa, no hay efecto estabilizador. ${ }^{18}$ Arimitsu y cols., en otro estudio anatómico, investigaron el papel que desempeña la membrana interósea en la estabilidad RCD, y hallaron un efecto estabilizador que tiene la osteotomía desde apenas un milímetro de acortamiento, cuando se realiza proximal a la inserción de la banda distal oblicua (presente en el 40\% de los especímenes utilizados). Asimismo, destacaron que la presencia de esta banda puede ser un factor de dificultad para realizar el acortamiento deseado e incluso conseguir la consolidación. ${ }^{19}$

El aumento de la tensión ligamentaria del borde cubital de la muñeca debido a un acortamiento cubital tiene dos efectos directos. Por un lado, el efecto estabilizador ya mencionado y, por el otro, un aumento de la presión a nivel de la articulación RCD que podría ser generador de dolor y artrosis en el futuro. Este aumento de la presión también se correlacionaría con la incongruencia articular provocada por el acortamiento.

Isa y cols. realizaron un estudio biomecánico cadavérico en el que evaluaron, en forma dinámica, los cambios de presión en la articulación cubitocarpiana con diferentes grados de acortamiento y en diferentes posiciones (flexión, desviación cubital, lanzador de dardos y pronación). Concluyeron en que, en la ICC idiopática (no asociada a fractura), $2 \mathrm{~mm}$ de acortamiento es una medida suficiente para descomprimir la articulación cubitocarpiana, sin generar fuerzas de tracción excesivas por la distensión ligamentaria.

Tatebe y cols. han evaluado la evolución de lesiones del disco articular del FCT detectadas por artroscopia durante una osteotomía de acortamiento y luego de varios años, mediante una segunda exploración artroscópica. El 50\% de las lesiones había cicatrizado, y las lesiones circulares tenían mayor potencial que las lineales. Por lo tanto, la descarga del disco articular y la estabilidad RCD obtenida tras la osteotomía de acortamiento facilitarían este proceso cicatricial.

Las ventajas de la osteotomía diafisaria de acortamiento cubital son: descomprime efectivamente la articulación cubitocarpiana, es un procedimiento extrarticular que preserva y retensa los ligamentos radiocubitales y cubitocarpianos, restituyendo la estabilidad RCD y cubitocarpiana; conserva el cartílago articular de la cúpula cubital y, en las secuelas de fracturas de radio sin deformidad sagital, restituye la anatomía RCD.

Las desventajas son el retraso o la falta de consolidación que oscila en un $4 \%$, que se han relacionado con el tabaquismo y que mejora notablemente con sistemas de acortamiento específico que permiten una muy buena estabilidad y compresión del foco de osteotomía. Asimismo, la tasa de retiro del implante por irritación local oscila entre el $11 \%$ y el $44 \%$, según diferentes publicaciones. Esta irritación local está determinada por la superficie del cúbito en la que se coloca el implante, la superficie volar es la que mejor tolerancia ha demostrado. El aumento de la presión transarticular RCD conllevaría el riesgo potencial de generar artrosis, sobre todo, en la forma oblicua invertida descrita por Tolat. ${ }^{16}$

Para evaluar las contraindicaciones de la osteotomía de acortamiento, debemos prestar atención a la articulación RCD. La artrosis, la deformidad sagital de la cavidad sigmoidea menor del radio como secuela de fractura, la configuración oblicua inversa de la clasificación de Tolat recién mencionada o la necesidad de un acortamiento excesivo constituyen las principales contraindicaciones.

\section{Procedimiento de resección en oblea (wafer)}

En 1992, Feldon describió este procedimiento para el tratamiento de las lesiones del FCT asociadas a ICC. Se basa en la resección del cartílago y el hueso subcondral del domo cubital (entre 2 y $4 \mathrm{~mm}$ de espesor), preservando las inserciones ligamentarias en la fóvea y la estiloides cubital y la articulación RCD. El objetivo es descomprimir la articulación cubitocarpiana y el disco articular del FCT (Figura 7). ${ }^{20}$ 


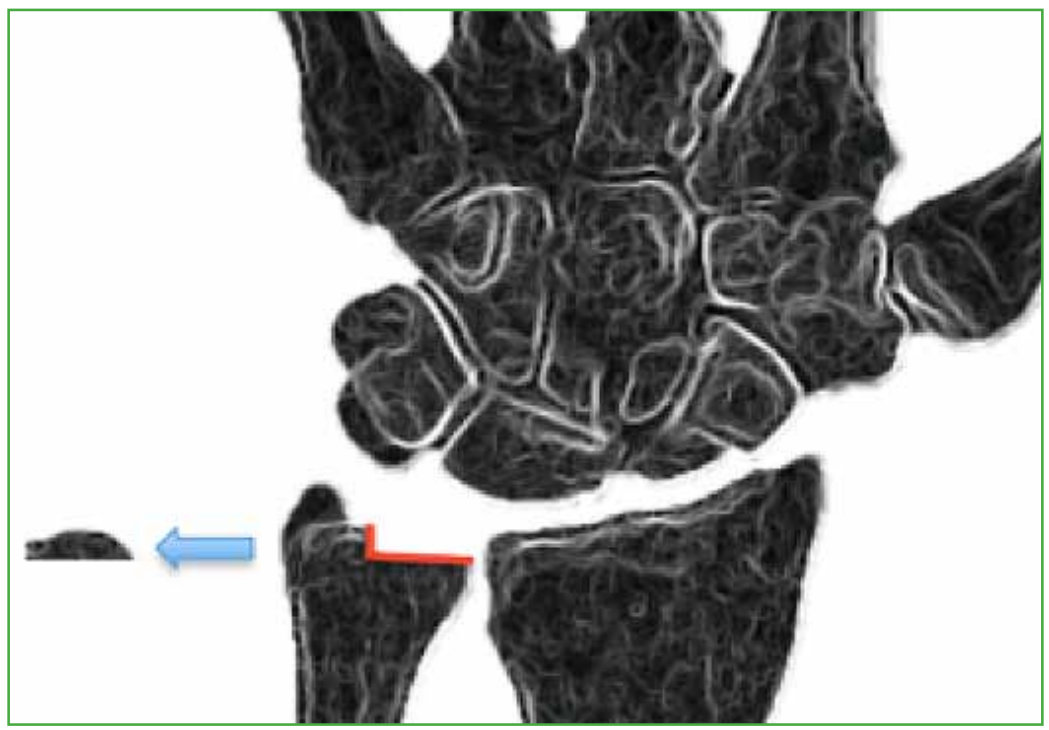

Figura 7. Procedimiento de la oblea (wafer).

Wnorowsky y cols., basándose en sus estudios cadavéricos, propusieron realizar este procedimiento de forma completamente artroscópica. Por medio de una perforación en el disco central del FCT, se introduce una fresa motorizada con la cual se reseca el cartílago del domo cubital y el hueso subcondral hasta conseguir un acortamiento de 2-3 mm. En publicaciones posteriores sobre esta técnica artroscópica, se ha recomendado utilizar el control radioscópico intraoperatorio para verificar la cantidad de hueso subcondral resecado, debido a la poca visualización. Tomaino y Weiser trataron a un grupo de 12 pacientes con lesiones del FCT (traumáticas y degenerativas) e ICC mediante un desbridamiento del disco articular del FCT más el procedimiento de la oblea (wafer) artroscópico. Todos los resultados fueron satisfactorios en cuanto al alivio del dolor. ${ }^{21}$

Las ventajas de este procedimiento son: descomprime la articulación cubitocarpiana, no requiere un período de consolidación ni material de osteosíntesis, el abordaje quirúrgico es limitado y permite tratar, a la vez, lesiones del FCT. Se puede realizar en forma mínimamente invasiva si se opta por la técnica artroscópica. Al no tensar ligamentos no aumenta la presión transarticular RCD, por lo cual no predispone a la aparición de artrosis. Se puede realizar en una configuración oblicua inversa de Tolat.

Las desventajas son: genera una lesión irreversible del cartílago articular del polo del cúbito, técnicamente es más demandante que una osteotomía diafisaria, la magnitud del acortamiento es limitada a 3 mm, el tiempo de recuperación es prolongado para lograr el alivio del dolor (3-6 meses), al no retensar ligamentos, no corrige inestabilidades RCD ni lunopiramidal, puede generar una hemartrosis con potencial artrofibrosis y no resuelve la impactación estilocarpiana. ${ }^{22}$ La técnica artroscópica, si bien es mininvasiva, no deja ver adecuadamente la cantidad de hueso subcondral resecado.

\section{Osteotomías metafisarias con abordaje articular}

En 2007, Slade y Gillon propusieron una técnica de osteotomía de acortamiento en cuña resectiva, de ubicación metafisaria subcondral, exponiendo la articulación RCD que luego fijan con dos tornillos canulados sin cabeza. ${ }^{23}$ Hammert y cols. describieron, con más detalle, esta misma técnica, pero utilizando solo un tornillo canulado y prefieren llamarla osteotomía de acortamiento cubital metafisaria distal, ya que describe con más precisión el sitio anatómico involucrado (Figura 8).

Barry y Macksoud describieron una técnica que consiste en una osteotomía resectiva subcondral metafisaria muy parecida a la que propuso Slade, pero incompleta, dejando la cortical más cubital intacta, para luego fracturarla en forma controlada, obteniendo así un acortamiento que, por su estabilidad, no requiere osteosíntesis (Figura 9). ${ }^{24}$ 


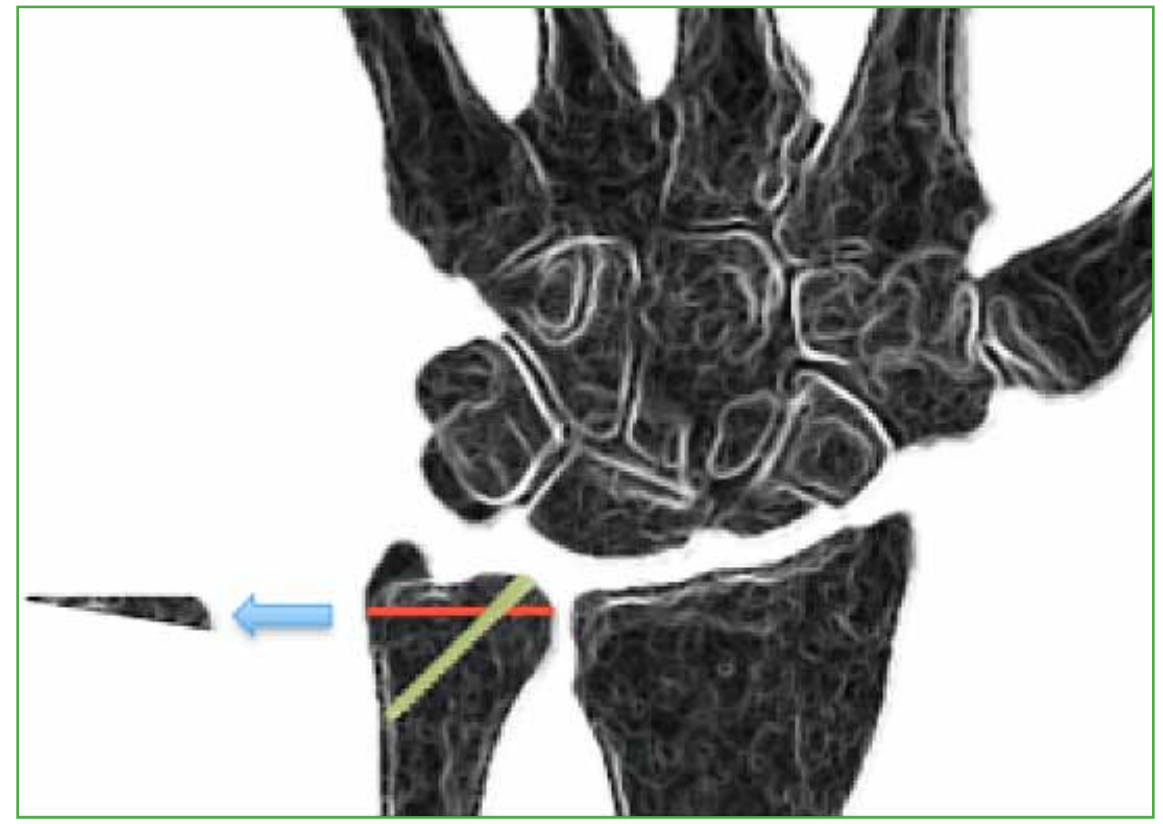

Figura 8. Osteotomía subcondral de Slade modificada por Hammert.

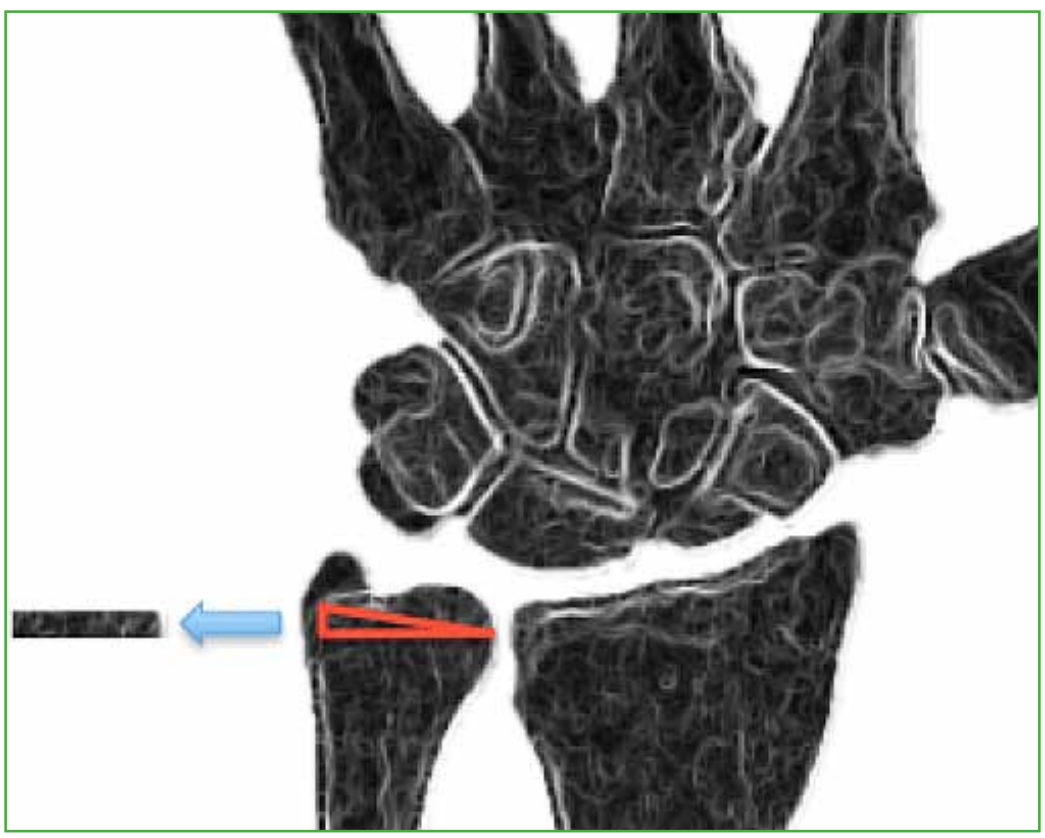

Figura 9. Osteotomía subcondral de Barry. 
En todas estas técnicas, se preserva el cartílago articular de la cúpula cubital, de la articulación RCD y las inserciones ligamentarias del FCT. A su vez, buscan un sitio de osteotomía con mayor potencial de consolidación. Refieren que sus pacientes han tenido escaso dolor posoperatorio, por lo cual los movilizan precozmente y han conseguido una excelente movilidad en un período de dos meses. En contraposición a las osteotomías diafisarias clásicas, estos autores destacan que el abordaje es limitado y que no necesitan de una segunda cirugía para retirar el material de osteosíntesis.

Las desventajas de estas técnicas con artrotomía serían que la cicatrización capsular puede generar una rigidez articular y que es una técnica demandante por la gran precisión manual que requiere la osteotomía para evitar consolidaciones viciosas..$^{24}$

Greenberg y cols. llevaron a cabo un estudio cadavérico para evaluar la biomecánica de este tipo de osteotomías metafisarias distales y concluyeron en que efectivamente disminuyen la carga cubitocarpiana en evaluaciones estáticas y dinámicas. También comprobaron que, a diferencia de las osteotomías diafisarias, el cambio de la geometría y la posición de la cabeza cubital no incrementaron las fuerzas de presión en la articulación RCD y, por lo tanto, no generarían artrosis..$^{25}$

\section{Osteotomía metafisaria sin abordaje articular}

Nunez y cols. proponen una osteotomía transversal subcapital sin la necesidad de exponer la articulación RCD. La estabilizan con una placa de osteosíntesis de cúbito distal bloqueada de bajo perfil, con ganchos distales que permiten tomar la estiloides cubital y realizar una compresión adecuada del sitio de la osteotomía. Por su bajo perfil, generaría menos conflicto con las partes blandas circundantes, menos prominencia cutánea y, por lo tanto, menor necesidad de una segunda cirugía para retirarla (Figura 10).

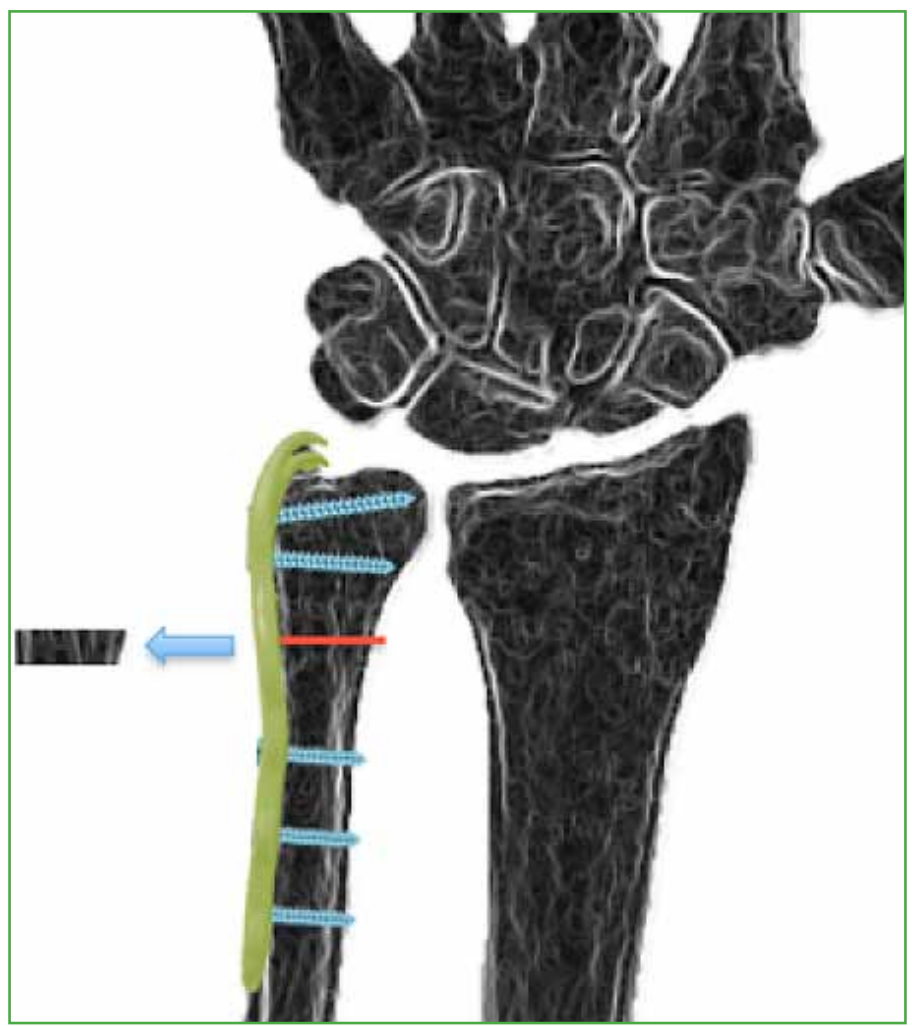

Figura 10. Osteotomía metafisaria subcapital de Nunez. 
Este procedimiento tiene el efecto de retensado ligamentario del FCT estabilizando las articulaciones RCD y lunopiramidal. Pero, por su ubicación anatómica, no interfiere con la tensión de la banda distal oblicua de la membrana interósea.

El sitio anatómico donde se realiza, por su irrigación y características óseas, tiene mejor potencial de consolidación y menos riesgo de osteonecrosis por alta temperatura que una osteotomía diafisaria convencional. ${ }^{6}$

\section{Direcciones futuras}

Se plantea el interrogante sobre cuál de todas estas técnicas es la mejor y la que se debería utilizar para una ICC. Creemos que no existe una técnica ideal para tratar todos los casos de esta enfermedad, por lo que, en primera instancia, proponemos su tipificación según el siguiente cuadro para orientar la mejor forma de tratamiento (Tabla 2).

Tabla 2. Tipificación de la impactación cubitocarpiana

\begin{tabular}{l|l}
$\begin{array}{l}\text { Característica } \\
\text { de la impactación } \\
\text { cubitocarpiana }\end{array}$ & Implicancia en el tratamiento \\
\hline Artrosis RCD & $\begin{array}{l}\text { La artrosis RCD avanzada es una contraindicación para realizar cualquier tipo de acortamiento } \\
\text { cubital y se debe pensar en procedimientos de rescate }\end{array}$ \\
\hline $\begin{array}{l}\text { Magnitud de la } \\
\text { discrepancia RCD }\end{array}$ & $\begin{array}{l}\text { Si es }>3 \text { mm, contraindica el procedimiento de la oblea (wafer), abierto o artroscópico, tampoco } \\
\text { se puede realizar una osteotomía sustractiva como han descrito Slade y Barry }\end{array}$ \\
\hline $\begin{array}{l}\text { Deformidad sagital } \\
\text { del radio }\end{array}$ & $\begin{array}{l}\text { En secuelas de fracturas del radio distal, si hay una deformidad en la orientación de la carilla } \\
\text { sigmoidea menor del radio, se debe pensar en una osteotomía del radio, ya que un acortamiento } \\
\text { cubital corregiría la discrepancia, pero generaría una incongruencia articular RCD }\end{array}$ \\
\hline $\begin{array}{l}\text { Inestabilidad RCD } \\
\text { o lunopiramidal }\end{array}$ & $\begin{array}{l}\text { Las osteotomías de acortamiento cubital diafisarias y metafisarias subcapitales tienen la } \\
\text { propiedad de recuperar estabilidad por retensado ligamentario si la inserción foveal está } \\
\text { conservada }\end{array}$ \\
\hline $\begin{array}{l}\text { Configuración según } \\
\text { Tolat }\end{array}$ & $\begin{array}{l}\text { La configuración oblicua inversa de Tolat es una contraindicación para las osteotomías diafisarias } \\
\text { y metafisarias subcapitales, porque podrían aumentar considerablemente las presión RCD y } \\
\text { empeorar el cuadro de dolor }\end{array}$ \\
\hline $\begin{array}{l}\text { Demanda funcional } \\
\text { Un procedimiento de la oblea (wafer) dejará una superficie cruenta e irregular por debajo del }\end{array}$ \\
\hline $\begin{array}{l}\text { Tabaquismo } \\
\text { RCD = radiocubital distal, FCT }=\end{array}$ & $\begin{array}{l}\text { El tabaque lo terminará dañando ante tareas repetitivas de esfuerzo. La conservación del cartílago } \\
\text { fel domo cubital es fundamental para seguir teniendo un deslizamiento con la fricción mínima }\end{array}$ \\
\hline
\end{tabular}

Consideramos que, a futuro, deberían realizarse estudios prospectivos, aleatorizados, multicéntricos, en grupos de pacientes con características definidas, que comparen la eficacia y las complicaciones de las diferentes técnicas quirúrgicas que hemos descrito. De esta forma, podríamos delinear, con mayor rigor científico, el lugar exacto que le corresponde a cada una de ellas.

Conflicto de intereses: Los autores no declaran conflictos de intereses. 


\section{BIBLIOGRAFÍA}

1. Linscheid RL. Biomechanics of the distal radioulnar joint. Clin Orthop Relat Res 1992;(275):46-55. PMID:1735232

2. Palmer AK, Werner FW. Biomechanics of the distal radioulnar joint. Clin Orthop Relat Res 1984;(187):26-35. PMID:6744728

3. Nakamura R, Tanaka Y, Imaeda T, Miura T. The influence of age and sex on ulnar variance. J Hand Surg Br 1991;16(1):84-8. https://doi.org/10.1016/0266-7681(91)90136-c

4. Orbay JL, Levaro-Pano F, Vernon LL, Cronin MH, Orbay JA, Tremols EJ. The parallelogram effect: the association between central band and positive ulnar variance. J Hand Surg Am 2018;43(9):827-32. https://doi.org/10.1016/j.jhsa.2018.03.058

5. Werner FW, Palmer AK, Fortino MD, Short WH. Force transmission through the distal ulna: effect of ulnar variance, lunate fossa angulation, and radial and palmar tilt of the distal radius. J Hand Surg Am 1992;17(3):423-8. https://doi.org/10.1016/0363-5023(92)90342-m

6. Nunez FA Jr, Barnwell J, Zhongyu L, Nunez FA Sr. Metaphyseal ulnar shortening osteotomy for the treatment of ulnocarpal abutment syndrome using distal ulna hook plate: case series. J Hand Surg Am 2012;37(8):1574-9. https://doi.org/10.1016/j.jhsa.2012.04.034

7. Friedman SL, Palmer AK. The ulnar impaction syndrome. Hand Clin 1991;7(2):295-310. PMID: 1880164

8. Cerezal L, del Piñal F, Abascal F. MR imaging findings in ulnar-sided wrist impaction syndromes. Magn Reson Imaging Clin North Am 2004;12(2):281-99. https://doi.org/10.1016/j.mric.2004.02.005

9. Minami A, Kato H. Ulnar shortening for triangular fibrocartilage complex tears associated with ulnar positive variance. J Hand Surg Am 1998;23(5):904-8. https://doi.org/10.1016/S0363-5023(98)80171-8

10. Barbaric K, Rujevcan G, Labas M, Delimar D, Bicanic G. Ulnar shortening osteotomy after distal radius fracture malunion: review of literature. Open Orthop J 2015;9(1):98-106. https://doi.org/10.2174/1874325001509010098

11. Owens J, Compton J, Day M, Glass N, Lawler E. Nonunion rates among ulnar-shortening osteotomy for ulnar impaction syndrome: a systematic review. J Hand Surg Am 2019;44(7):612.e1-612.e12.

https://doi.org/10.1016/j.jhsa.2018.08.018

12. Tomaino MM, Elfar J. Ulnar impaction syndrome. Hand Clin 2005;21(4):567-75. https://doi.org/10.1016/j.hcl.2005.08.011

13. Nakamura R, Horii E, Imaeda T, Nakao E, Kato H, Watanabe K. The ulnocarpal stress test in the diagnosis of ulnarsided wrist pain. J Hand Surg Br 1997;22(6):719-23. https://doi.org/10.1016/s0266-7681(97)80432-9

14. Baek GH, Chung MS, Lee YH, Gong HS, Lee S, Kim HH. Ulnar shortening osteotomy in idiopathic ulnar impaction syndrome. Surgical technique. J Bone Joint Surg Am 2006;88(1):212-20. https://doi.org/10.2106/JBJS.F.00320

15. McBeath R, Katolik LI, Shin EK. Ulnar shortening osteotomy for ulnar impaction syndrome. J Hand Surg Am 2013;38(2):379-81. https://doi.org/10.1016/j.jhsa.2012.07.019

16. Tatebe M, Nishizuka T, Hirata H, Nakamura R. Ulnar shortening osteotomy for ulnar-sided wrist pain. J Wrist Surg 2014;3(2):77-4. https://doi.org/10.1055/s-0034-1372516

17. Baek GH, Lee HJ, Gong HS, Rhee SH, Kim J, Kim KW, et al. Long-term outcomes of ulnar shortening osteotomy for idiopathic ulnar impaction syndrome: at least 5-years follow-up. Clin Orthop Surg 2011;3(4):295-301. https://doi.org/10.4055/cios.2011.3.4.295

18. Nishiwaki M, Nakamura T, Nakao Y, Nagura T, Toyama Y. Ulnar shortening effect on distal radioulnar joint stability: a biomechanical study. J Hand Surg Am 2005;30(4):719-26. https://doi.org/10.1016/j.jhsa.2005.04.015

19. Arimitsu S, Moritomo H, Kitamura T, Berglund LJ, Zhao K, An K, et al. The stabilizing effect of the distal interosseous membrane on the distal radioulnar joint in an ulnar shortening procedure: a biomechanical study. $J$ Bone Joint Surg Am 2011;93(21):2022-30. https://doi.org/10.2106/JBJS.J.00411

20. Feldon P, Terrono AL, Belsky MR. Wafer distal ulna resection for triangular fibrocartilage tears and/or ulna impaction syndrome. J Hand Surg Am 1992;17(4):731-7. https://doi.org/10.1016/0363-5023(92)90325-j

21. Tomaino MM, Weiser RW. Combined arthroscopic TFCC debridement and wafer resection of the distal ulna in wrists with triangular fibrocartilage complex tears and positive ulnar variance. J Hand Surg Am 2001;26(6):1047-52. https://doi.org/10.1053/jhsu.2001.28757

22. Griska A, Feldon P. Wafer resection of the distal ulna. J Hand Surg Am 2015;40(11):2283-8. https://doi.org/10.1016/j.jhsa.2015.08.018 
23. Slade JF, Gillon TJ. Osteochondral shortening osteotomy for the treatment of ulnar impaction syndrome: a new technique. Tech Hand Up Extrem Surg 2007;11(1):74-82. https://doi.org/10.1097/bth.0b013e3180337df9

24. Barry JA, Macksoud WS. Cartilage-retaining wafer resection osteotomy of the distal ulna. Clin Orthop Relat Res 2008;466(2):396-401. https://doi.org/10.1007/s11999-007-0059-5

25. Greenberg JA, Werner FW, Smith JM. Biomechanical analysis of the distal metaphyseal ulnar shortening osteotomy. J Hand Surg Am 2013;38(10):1919-24. https://doi.org/10.1016/j.jhsa.2013.06.038 